\author{
Journal of \\ Materials Chemistry B
}

\title{
Effects of nanocrystalline hydroxyapatites on macrophage polarization
}

\begin{tabular}{|r|l|}
\hline Journal: & Journal of Materials Chemistry B \\
\hline Manuscript ID & Draft \\
\hline Article Type: & Paper \\
\hline Date Submitted by the Author: & $\mathrm{n} / \mathrm{a}$ \\
\hline Complete List of Authors: & $\begin{array}{l}\text { Linares, Javier; Universidad Complutense de Madrid, Bioquímica y Biología } \\
\text { Molecular I } \\
\text { Fernández, Ana Belén; Universidad Complutense de Madrid, Departamento } \\
\text { de Bioquímica y Biología Molecular I } \\
\text { Feito, María José; Universidad Complutense de Madrid, Bioquímica y } \\
\text { Biología Molecular I } \\
\text { Matesanz, Concepción; Universidad Complutense de Madrid, Departamento } \\
\text { de Bioquímica } \\
\text { Sánchez-Salcedo, Sandra; Universidad Complutense, Quimica Inorgánica y } \\
\text { Bioinorgánica } \\
\text { Arcos, Daniel; Universidad Complutense de Madrid, } \\
\text { Vallet-Regi, Maria; Universidad Complutense de Madrid, Spain, } \\
\text { Rojo, Jose María; Centro de Investigaciones Biológicas, CSIC, } \\
\text { Portolés, María Teresa; Universidad Complutense de Madrid, Departamento } \\
\text { de Bioquímica }\end{array}$ \\
\hline
\end{tabular}


Madrid, 5th January 2016

\section{Dear Editor:}

I am pleased to submit the manuscript "Effects of nanocrystalline hydroxyapatites on macrophage polarization" authored by Javier Linares, Ana Belén Fernández, María José Feito, María Concepción Matesanz, Sandra Sánchez-Salcedo, Daniel Arcos, María Vallet-Regí, José María Rojo and María Teresa Portolés, to be considered for publication in Journal of Materials Chemistry B.

The present study is focused on the effects of nanocrystalline hydroxyapatite (nano-HA) and nanocrystalline silicon substituted hydroxyapatite (nano-SiHA) on the macrophage populations defined as proinflammatory (M1) and reparative (M2) phenotypes. The results demonstrate that both nano-HA and nano-SiHA favour the macrophage polarization towards a M2 reparative phenotype, decreasing M1 population and ensuring an appropriate response in the implantation site of these biomaterials.

We believe that this work will have an enormous interest for the use of these nanocrystalline hydroxyapatites for bone repair and bone tissue engineering.

All the co-authors have read and approved the manuscript and consent in submitting it to this journal for consideration. The results presented are unpublished and are not under consideration for publication in any other journal.

Thanking you very much for your attention, I remain

Sincerely yours

M. Teresa Portolés

\section{Correspondence address:}

Professor M.T. Portolés. Departamento de Bioquímica y Biología Molecular I, Facultad de Ciencias Químicas, Universidad Complutense, 28040-Madrid, Spain.

FAX: 34-1-394 4159

E-mail: portoles@quim.ucm.es 


\title{
Journal Name
}

\section{ARTICLE}

\section{Effects of nanocrystalline hydroxyapatites on macrophage polarization}

Received 00th January 20xx, Accepted 00th January 20xx

DOI: $10.1039 / x 0 x \times 00000 x$

www.rsc.org/

\author{
J. Linares, ${ }^{a}$ A. B. Fernández, ${ }^{a}$ M. J. Feito, ${ }^{a, b}$ M. C. Matesanz, ${ }^{a}$ S. Sánchez-Salcedo, ${ }^{c, d}$ D. Arcos, ${ }^{c, d}$ M. \\ Vallet-Regí, ${ }^{c, d}$ J. M. Rojo ${ }^{e}$ and M. T. Portolés ${ }^{a, b^{*}}$
}

\begin{abstract}
Silicon substituted and nanocrystalline hydroxyapatites have attracted the attention of many researchers due to their upregulation in osteoblast cell metabolism and enhanced bioreactivity, respectively. On the other hand, the biomaterial success or failure depends ultimately on the immune response triggered after its implantation. Macrophages are the main components of the innate immune system with an important role in healing and tissue remodelling due to their remarkable functional plasticity, existing a whole spectrum of functional populations with varying phenotypic features. The effects of nanocrystalline hydroxyapatite (nano-HA) and nanocrystalline silicon substituted hydroxyapatite (nano$\mathrm{SiHA}$ ) on the macrophage populations defined as proinflammatory (M1) and reparative (M2) phenotypes, have been evaluated in the present study with RAW 264.7 cells and mouse peritoneal macrophages as in vitro models. With the purpose of favouring the polarization of primary macrophages towards the M1 or M2 phenotype, the pro-inflammatory stimulus LPS or the anti-inflammatory stimulus IL-10 were used respectively, evaluating the biomaterial effects under these conditions. Our results show that both nano-HA and nano-SiHA favour the macrophage polarization towards a M2 reparative phenotype, decreasing M1 population and ensuring an appropriate response in the implantation site of these biomaterials designed for bone repair and bone tissue engineering.
\end{abstract}

\section{Introduction}

The success or failure of a biomaterial depends ultimately on the immune response triggered after its implantation. Macrophages are the main defence components of innate immune system also including dendritic cells, granulocytes, mast cells and natural killer cells. ${ }^{1}$ Recently, the role of macrophages in healing and tissue remodelling after injury has been demonstrated that is due to their remarkable functional plasticity and capability to polarize into classically activated / inflammatory (M1) and alternatively activated / reparative (M2) phenotypes..$^{2-5}$ These two states represent the extremes of a whole spectrum of populations characterized by the expression of distinct cell surface markers, secreted cytokines, transcription factors and epigenetic pathways. ${ }^{2,6,7}$ The capacity of macrophages to play both positive and negative roles in disease processes and tissue repair depends on the M1/M2 balance. ${ }^{5}$ Thus, the identification of mechanisms underlying functional polarization of macrophages into $\mathrm{M} 1$ or $\mathrm{M} 2$ cells

\footnotetext{
a. Department of Biochemistry and Molecular Biology I/Faculty of Chemistry, Universidad Complutense de Madrid, Spain.

${ }^{b}$ Instituto de Investigación Sanitaria San Carlos IdISSC, Spain

Department of Inorganic and Bioinorganic Chemistry/Faculty of Pharmacy, Universidad Complutense de Madrid, Instituto de Investigación Hospital 12 de Octubre i+12, Spain

${ }^{d .}$ Networking Research Center on Bioengineering/Biomaterials and Nanomedicine CIBER-BBN, Spain

e. Department of Cellular and Molecular Medicine/Centro de Investigaciones Biológicas, CSIC, Spain
}

constitutes the base for macrophage-centered diagnostic and therapeutic strategies for multiple diseases. ${ }^{3}$ Concerning normal tissue repair, macrophages exhibit a pro-inflammatory phenotype (M1) at early stages, involved in angiogenesis initiation, and a pro-healing phenotype (M2) at later stages, promoting vessel maturation. ${ }^{8}$ In this context, new biomaterials are prepared with the aim to polarize selectively macrophages in order to obtain appropriate responses contributing to successful tissue integration. ${ }^{4,9}$ In this regard, sequential delivery of immunomodulatory cytokines to promote the $\mathrm{M} 1$ to $\mathrm{M} 2$ transition of macrophages, enhancing vascularization of bone scaffolds, has been recently proposed. ${ }^{8}$ Due to its similarity to the bone tissue mineral, synthetic hydroxyapatite (HA) is a calcium phosphate ceramic widely used in the field of dentistry and orthopaedic surgery with different purposes as grafting for small bone defects, coatings of metallic prostheses and periodontal implants, and as scaffolds for bone tissue engineering. ${ }^{10}$ Silicon substituted hydroxyapatite ( $\mathrm{SiHA}$ ) presents better bioactivity in vivo than $\mathrm{HA}^{11}$ and has attracted the attention of many researchers. ${ }^{12-16}$ The presence of silicate within the crystalline structure produces a higher solution-mediated degradation of the apatite phase at the grain boundary and an up-regulation in osteoblast cell metabolism in the early stages of bone formation. ${ }^{13,15,17}$ For these reasons, SiHA has recently been incorporated to the biomaterials market as Actifuse ABXTM (Apatech Ltd, UK) for spinal, orthopedic, periodontal, oral and craniomaxillofacial applications. SiHA approved for clinical use 
are highly crystalline bioceramics treated at high temperatures. However, the possibility of enhancing the bioceramics bioreactivity through their preparation with low temperature methods has been suggested. ${ }^{18,19}$ Avoiding the high temperature sintering process (commonly about 1200 ㅇ) , nanocrystalline pieces and grains can be prepared. Higher surface area and smaller crystal size could thus provide very interesting bioresponses, especially in $\mathrm{SiHA}$ as the osteogenic effect of silicon is mainly explained by its location at the crystal boundaries. $^{12,13}$

We recently showed that both nanocrystalline hydroxyapatite (nano-HA) and nanocrystalline silicon substituted hydroxyapatite (nano-SiHA) produced a significant proliferation decrease of RAW-264.7 cells (a mouse macrophage cell line retaining many of the characteristics of macrophages in vivo) in comparison with controls in the absence of material. ${ }^{20}$ This effect has been also observed with cultured 1929 fibroblasts, Saos-2 osteoblasts and MC3T3-E1 preosteoblasts. ${ }^{14,21}$ On the other hand, nano-SiHA disks delayed the osteoclast differentiation and decreased the resorptive activity of these cells on their surface, as compared to nano-HA samples, without affecting cell viability, ${ }^{22}$ thus indicating a beneficial action of Si-substituted material for bone regeneration in agreement with other studies. ${ }^{13,15,17,23,24}$ Since the balance between proinflammatory (M1) and reparative (M2) macrophages has been involved in their negative or positive role in disease and tissue remodelling, ${ }^{4,5,8,9}$ the effects of nano-HA and nano-SiHA on murine macrophage populations have been evaluated in the present study in basal conditions and in the presence of either pro-inflammatory (LPS) or anti-inflammatory (IL-10) stimuli.

\section{Experimental}

Nanocrystalline hydroxyapatite and silicon substituted hydroxyapatite synthesis. Samples of pure and silicon substituted $\mathrm{HA}$ were prepared by aqueous precipitation reaction of $\mathrm{Ca}\left(\mathrm{NO}_{3}\right)_{2} \bullet 4 \mathrm{H}_{2} \mathrm{O}, \quad\left(\mathrm{NH}_{4}\right)_{2} \mathrm{HPO}_{4}$ and $\mathrm{Si}\left(\mathrm{CH}_{3} \mathrm{CH}_{2} \mathrm{O}\right)_{4}$ solutions. The amounts of reactants were calculated on the assumption that silicon would be substituted by phosphorus. Two different compositions have been prepared with nominal formula $\mathrm{Ca}_{10}\left(\mathrm{PO}_{4}\right)_{6-x}\left(\mathrm{SiO}_{4}\right)_{x}(\mathrm{OH})_{2-x}$, with $\mathrm{x}=0$ and 0.25 for nano-HA and nano-SiHA samples, respectively. $1 \mathrm{M}$ $\mathrm{Ca}\left(\mathrm{NO}_{3}\right)_{2} \bullet 4 \mathrm{H}_{2} \mathrm{O}$ solution was added to $\left(\mathrm{NH}_{4}\right)_{2} \mathrm{HPO}_{4}$ and TEOS solutions of stoichiometric concentration to obtain the compositions described above. The mixture was stirred for 12 hours at $80^{\circ} \mathrm{C}$. During the reaction the $\mathrm{pH}$ was continuously adjusted to 9.5 to ensure constant conditions during the synthesis.

Fractions of $300 \mathrm{mg}$ of the as-precipitated powders of nano-HA and nano-SiHA materials were pressed into disk-shape $(11 \mathrm{~mm}$ diameter, $2 \mathrm{~mm}$ height) by means of 3 tons of uniaxial pressing. Thereafter the disks were treated at $700^{\circ} \mathrm{C}$ for two hours under air atmosphere to remove the nitrates without introducing important changes in the crystallite size respect to the as precipitated powder. Finally, one disk of each sample were milled and sieved. The HA and Si-HA grains thus obtained have a diameter ranging in size between 10 to 100 micrometers, as determined by means of a Sedigraph 5100 equipment (data not shown).

Chemical, structural and microstructural studies of nano-HA and nano-SiHA. Elemental chemical analysis was carried out by fluorescence X-ray spectrometry for $\mathrm{P}, \mathrm{Si}$ and $\mathrm{Ca}$. XRD patterns were collected with a Philips PW 1730 X-ray diffractometer using $\mathrm{Cu} \mathrm{K \alpha}$ radiation with a step size of $0.02 \quad 2 \theta^{\circ}$ and 8 seconds of counting time. In order to determine the crystalline and microstructural characteristic of both samples, Rietveld refinements were carried out over the XRD patterns collected. The refinements were performed using the atomic position set and the space group of the HA structure $\mathrm{P}_{3} / \mathrm{m}$, No. $176^{25,26}$ The instrumental resolution function (IRF) of the diffractometer was obtained from a very-well-crystallized hydroxylapatite sample and taken into account in a separate input file. Textural properties (surface area, pore volume and pore size) were determined by $\mathrm{N}_{2}$ adsorption porosimetry with a Micromeritics ASAP 2020 equipment. To perform the $\mathrm{N}_{2}$ measurements, the samples were previously degassed under vacuum for $24 \mathrm{~h}$ at $80 \stackrel{\circ}{ } \mathrm{C}$. Zeta potential was measured by means of a Zetasizer Nano ZS (Malvern Instruments).

Culture of RAW-264.7 macrophages. RAW-264.7 cells were seeded in 6 well culture plates (CULTEK S.L.U., Madrid, Spain), at a density of either $10^{5}$ cells $/ \mathrm{ml}$, in $2 \mathrm{ml}$ of Dulbecco's Modified Eagle Medium (DMEM) supplemented with $10 \%$ fetal bovine serum (FBS, Gibco, BRL), $1 \mathrm{mM}$ L-glutamine (BioWhittaker Europe, Belgium), penicillin $(200 \mu \mathrm{g} / \mathrm{ml}$, BioWhittaker Europe, Belgium), and streptomycin $(200 \mu \mathrm{g} / \mathrm{ml}$, BioWhittaker Europe, Belgium) at $37^{\circ} \mathrm{C}$ under a $\mathrm{CO}_{2}$ (5\%) atmosphere. After 1 day or 3 days culture in the presence or the absence of $1 \mathrm{mg} / \mathrm{ml}$ of either nano-HA or nano-SiHA, the attached RAW-264.7 cells were washed with phosphate buffered saline (PBS) and harvested using cell scrapers. Then, cell suspensions were centrifuged at $310 \times \mathrm{xg}$ for $10 \mathrm{~min}$ and treated with different antibodies in order to characterize the macrophage phenotypes by flow cytometry as described below.

Isolation and culture of murine peritoneal macrophages. Normal macrophages were obtained from the peritoneum of untreated mice as described elsewhere. ${ }^{27}$ Briefly, BALB/c mice were killed, and the skin was removed from the abdominal area. Mice were then injected intraperitoneally with 4-5 $\mathrm{ml}$ of phosphate buffered saline (PBS) using an 18 gauge needle. Without extracting the needle, the abdomen was gently massaged and then as much fluid from the peritoneum as possible was slowly withdrawn with the syringe. After removing, the peritoneal cells were gently washed with PBS before use. All procedures were approved by Institutional Animal Care and Use Committees. Mouse peritoneal macrophages were seeded on either nano-HA or nano-SiHA disks, previously introduced into 24 well culture plates 
(CULTEK S.L.U., Madrid, Spain), at a density of $10^{5}$ cells $/ \mathrm{ml}$ in 1 $\mathrm{ml}$ of Minimum Essential Medium Alpha ( $\alpha$-MEM) supplemented with $10 \%$ fetal bovine serum (FBS, Gibco, BRL), $1 \mathrm{mM}$ L-glutamine (BioWhittaker Europe, Belgium), penicillin (200 $\mathrm{\mu g} / \mathrm{ml}$, BioWhittaker Europe, Belgium), and streptomycin (200 $\mathrm{\mu g} / \mathrm{ml}$, BioWhittaker Europe, Belgium) under a $\mathrm{CO}_{2}(5 \%)$ atmosphere and at $37 \circ \mathrm{C}$. After $2 \mathrm{~h}$, the culture medium was renewed to remove non-adherent cells (mostly $B$ and $T$ lymphocytes). Then, peritoneal macrophages were cultured for 3 days in the presence of either $E$. coli lipopolysaccharide (LPS, $25 \mu \mathrm{g} / \mathrm{ml}$, Sigma-Aldrich Corporation, St. Louis, MO, USA) or interleukin-10 (IL-10, 20 ng/ml, Sigma-Aldrich Corporation, St. Louis, MO, USA) as pro-inflammatory and anti-inflammatory stimuli, respectively. ${ }^{2}$ Controls in the absence of materials and without stimuli were carried out in parallel. After 3 days culture, murine peritoneal macrophages were washed with PBS and detached with PBS / 1mM EDTA at 4 o $C$. Then, cell suspensions were centrifuged at 310xg for $10 \mathrm{~min}$ and treated with different antibodies in order to characterize M1 and M2 macrophage phenotypes by flow cytometry as described below.

Characterization of $\mathbf{M} 1$ and $\mathrm{M} 2$ macrophage phenotypes. $\mathrm{M} 1$ and M2 macrophage phenotypes were characterized by flow cytometry by the expression of CD80 and CD163, known M1 and M2 markers, respectively. ${ }^{28,29}$ After detachment and centrifugation, cells were incubated in $45 \mu$ of staining buffer (PBS, 2.5\% FBS Gibco, BRL and $0.1 \%$ sodium azide, SigmaAldrich Corporation, St. Louis, MO, USA) with $5 \mu$ l of normal mouse serum inactivated for $15 \mathrm{~min}$ at 4 o $\mathrm{C}$ in order to block the Fc receptors on the macrophage plasma membrane, before adding the primary antibody, and to prevent nonspecific binding. Then, to evaluate CD80 and CD163 expression, cells were incubated with either phycoerythrin $(\mathrm{PE})$ conjugated anti-mouse CD80 antibody $(2.5 \mu \mathrm{g} / \mathrm{ml}$, BioLegend, San Diego, California) or phycoerythrin (PE) conjugated antimouse CD163 (2.5 $\mu \mathrm{g} / \mathrm{ml}$, BioLegend, San Diego, California) for $30 \mathrm{~min}$ in the dark. Labelled cells were analyzed using a FACSCalibur flow cytometer. PE fluorescence was excited at $488 \mathrm{~nm}$ and measured at 585/42 nm. The conditions for the data acquisition and analysis were established using negative and positive controls with the CellQuest Program of Becton Dickinson and these conditions were maintained during all the experiments. Each experiment was carried out three times and single representative experiments are displayed. For statistical significance, at least 10,000 cells were analyzed in each sample and the mean of the fluorescence emitted by these single cells was used.

Morphological studies of M1 and M2 macrophages by Confocal Microscopy. Macrophages cultured on nano-HA and nano-SiHA disks were fixed with $3.7 \%$ paraformaldehyde (Sigma-Aldrich Corporation, St. Louis, MO, USA) in PBS for $10 \mathrm{~min}$, washed with PBS and permeabilized with $0.1 \%$ Triton X-100 (SigmaAldrich Corporation, St. Louis, MO, USA) for $5 \mathrm{~min}$. The samples were then washed with PBS and preincubated with PBS containing 1\% BSA (Sigma-Aldrich Corporation, St. Louis, MO, USA) for $30 \mathrm{~min}$ to prevent non-specific binding. Samples were incubated in $1 \mathrm{ml}$ of staining buffer with either phycoerythrin conjugated anti-mouse CD80 antibody (2.5 $\mu \mathrm{g} / \mathrm{ml}$, BioLegend, San Diego, California) or phycoerythrin conjugated anti-mouse CD163 $(2.5 \mu \mathrm{g} / \mathrm{ml}$, BioLegend, San Diego, California) for $30 \mathrm{~min}$ at $4{ }^{\circ} \mathrm{C}$ in the dark. Then, cells were incubated during 20 min with FITC-phalloidin (Dilution 1:40, Molecular Probes) to stain F-actin filaments. Samples were then washed with PBS and the cell nuclei were stained with DAPI (4'-6-diamidino-2'-phenylindole, $3 \mu \mathrm{M}$ in PBS, Molecular Probes) for $5 \mathrm{~min}$. Samples were examined by a LEICA SP2 Confocal Laser Scanning Microscope. The fluorescence of FITC was excited at $488 \mathrm{~nm}$ and the emitted fluorescence was measured at 491-586 nm. PE fluorescence was excited at $488 \mathrm{~nm}$ and measured at 575-675 $\mathrm{nm}$. DAPI fluorescence was excited at $405 \mathrm{~nm}$ and measured at $420-480$ $\mathrm{nm}$.

Inflammatory cytokine detection. The amounts of TNF- $\alpha$, and IL6 in the culture medium were quantified by ELISA (Gen-Probe, Diaclone), carried out according to the manufacturer's instructions.

Statistics. Data are expressed as means + standard deviations of one representative experiment out of three experiments carried out in triplicate. Statistical analysis was performed using the Statistical Package for the Social Sciences (SPSS) version 19 software. Statistical comparisons were made by analysis of variance (ANOVA). Scheffé test was used for post hoc evaluations of differences among groups. $\mathrm{P}<0.05$ was considered as statistically significant.

\section{Results and discussion}

Macrophages are the main innate immune cells involved in regulating the primary response to any stimuli including reactions to implanted biomaterials. In the present study, the effects of nanocrystalline hydroxyapatite (nano-HA) and nanocrystalline silicon substituted hydroxyapatite (nano-SiHA) on murine macrophage populations have been evaluated in basal conditions and in the presence of either proinflammatory (LPS) or anti-inflammatory (IL-10) stimuli.

Macrophages display plasticity and change their functional profiles in response to their environment exhibiting a spectrum of polarization states related to their functional diversity. ${ }^{2-4,30}$ At the extremes of this spectrum of populations, there are the activated/inflammatory (M1) and the alternatively activated/reparative (M2) states., ${ }^{5,31}$ The M1 phenotype results from macrophage interaction with granulocyte-macrophage colony stimulating factor (GM-CSF) and pro-inflammatory signals such as interferon- $\gamma$ (IFN- $\gamma$ ) and bacterial lipopolysaccharide (LPS). ${ }^{32}$ M1 macrophages are 
capable of antigen presentation, promote the differentiation of Th1 lymphocytes and produce pro-inflammatory cytokines (IL-12, IL-1, IL-6, IL-23, TNF- $\alpha$, and CXCL10), showing cytotoxic activity against microorganisms and neoplastic cells. ${ }^{33} \mathrm{M} 1$ cells also produce reactive oxygen species (ROS) which can damage neighboring cells increasing the pro-inflammatory response. ${ }^{34}$ Concerning the implantation of biomaterials, while the initial presence of $\mathrm{M} 1$ macrophages is necessary, a prolonged presence of these cells leads to chronic inflammatory events and failure of biomaterial integration. ${ }^{4}$ On the other hand, M2 macrophages is the result of stimulation by macrophage colony stimulating factor (M-CSF), IL-4, IL-13, IL-10 or immunosuppressive agents. $\mathrm{M} 2$ cells secrete antiinflammatory molecules (IL-10, CCL17, CCL22, CCL18, IL-1ra, and IL-1 decoy receptor), show a high scavenging activity and promote tissue repair and angiogenesis, favouring tumor progression. ${ }^{35} \mathrm{M} 2$ cells include different subsets: $M 2 a, M 2 b$, $\mathrm{M} 2 \mathrm{c}^{30,33} \mathrm{M} 2 \mathrm{a}$ (induced by IL-4 and IL-13) and M2b (induced by immune complexes and toll like receptor agonists) macrophages carry out immune regulatory functions by initiating Th2 lymphocyte anti-inflammatory responses. ${ }^{33} \mathrm{M} 2 \mathrm{c}$ macrophages (induced by IL-10) plays a key role in tissue remodeling and suppression of inflammatory immune response, $^{2}$ favouring the vascularization of regenerative biomaterials and their integration. ${ }^{4}$

To analyze the effect of nano-HA and nano-Si HA hydroxyapatites on $\mathrm{M} 1$ and $\mathrm{M} 2$ phenotypes, RAW-264.7 macrophages and mouse peritoneal macrophages were cultured either in the presence of $1 \mathrm{mg} / \mathrm{ml}$ of these powdered materials or on the surface of compact disks prepared with these nanocrystalline hydroxyapatites. After culture, macrophage phenotypes were characterized by flow cytometry through the expression of CD80 and CD163, known $\mathrm{M} 1$ and $\mathrm{M} 2$ markers, respectively. ${ }^{28,29}$

Nano-HA and nano-SiHA show chemical compositions very similar to the theoretical ones as deduced from the $\mathrm{Ca}$, and $\mathrm{Si}$ compositions shown in Table 1 . The molar ratio $\mathrm{Ca} / \mathrm{P}+\mathrm{Si}$ are in both cases very close to 1.67 , indicating that silicon substitutes for $P$ in nano-SiHA as previously reported elsewhere. ${ }^{16}$ The surface area (calculated as $\mathrm{S}_{\mathrm{BET}}$ ) porosity and pore size correspond to materials deficiently sintered as consequence of

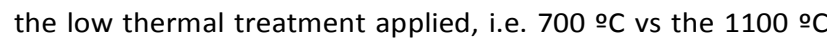
or more commonly used for the preparation of highly crystalline hydroxyapatites.

Table 1. Chemical, textural, microstructural and surface charge properties of nano-HA and nano-SiHA.

\begin{tabular}{|c|c|c|c|c|c|c|c|c|}
\hline \multirow[b]{2}{*}{ Sample } & \multicolumn{3}{|c|}{$\begin{array}{c}\text { Chemical composition } \\
\text { (\%wt) }\end{array}$} & \multicolumn{3}{|c|}{ Textural properties } & \multirow{2}{*}{$\begin{array}{c}\text { Crystallite } \\
\text { Size } \\
(\mathrm{nm})\end{array}$} & \multirow{2}{*}{$\begin{array}{c}\text { Zeta } \\
\text { Potential } \\
(\mathrm{mV})\end{array}$} \\
\hline & $\mathrm{Ca}$ & $P$ & $\mathrm{Si}$ & $\begin{array}{c}\mathrm{S}_{\mathrm{BET}} \\
\left(\mathrm{m}^{2} \cdot \mathrm{g}^{-1}\right)\end{array}$ & $\begin{array}{c}\text { Pore } \\
\text { volume } \\
\left(\mathrm{cm}^{3} \cdot \mathrm{g}^{-1}\right)\end{array}$ & $\begin{array}{c}\text { Pore } \\
\text { size } \\
(\mathrm{nm})\end{array}$ & & \\
\hline Nano-HA & 39.5 & 17.8 & 0.0 & 17.0 & 0.04 & 8.7 & 30 & -2.3 \\
\hline Nano-SiHA & 41.0 & 18.0 & 0.51 & 25.9 & 0.17 & 26.8 & 22 & -8.6 \\
\hline
\end{tabular}

The low thermal treatment results in pieces containing porosities and surface areas significantly higher than those obtained after treatments at high temperature. The crystallite sizes calculated by the refinement of XRD patterns show that both materials are formed by nanocrystallites (sizes below 100 $\mathrm{nm}$ ), evidencing that the $700 \stackrel{\circ}{\circ}$ used in this syntheses could not promote the crystal growth associated to the conventional solid state reaction at higher temperatures. Nano-SiHA shows higher textural parameters and smaller crystallite size, which is indicative of a higher number of microstructural defects in this solid. Beside, surface charge is lower in nano-SiHA, as could be expected when $\mathrm{PO}_{4}{ }^{3-}$ groups are substituted by $\mathrm{SiO}_{4}{ }^{4-}$ groups introducing an extra negative charge. Microstructural defects and lower surface charge are considered to play an important role in the improved behavior of silicon substituted hydroxyapatites respect to non-substituted ones. ${ }^{11,12}$

Figure 1 shows the effects of powdered nano-HA and nanoSiHA $(1 \mathrm{mg} / \mathrm{ml})$ on the percentage of $\mathrm{M} 1\left(\mathrm{CD}^{\circ} 0^{+}\right)$and $\mathrm{M} 2$ $\left(\mathrm{CD}_{163}{ }^{+}\right)$macrophage populations after 1 day of treatment of RAW 264.7 cells. Control cultures without material were also carried out in parallel. A balance of macrophage M1-M2 polarization was observed in control RAW 264.7 cells after 1 day of culture, obtaining similar percentages of both M1 and M2 macrophages, around $40 \%$. The presence of powdered nano-HA and nano-SiHA induced a significant decrease of these extreme populations in comparison with control macrophages. No significant differences were observed between the effects of nano-HA and nano-SiHA on $\mathrm{M} 1\left(\mathrm{CD} 80^{+}\right)$ population. However, the $\mathrm{M} 2\left(\mathrm{CD}_{163}{ }^{+}\right)$population was significantly higher with nano-SiHA than with nano-HA after 1 day.

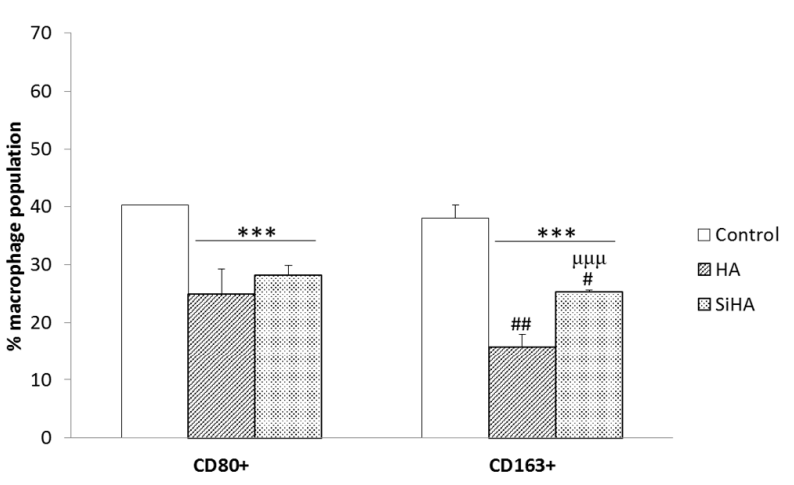

Figure 1. Effects of powdered nano-HA and nano-SiHA (1 $\mathrm{mg} / \mathrm{ml})$ on $\mathrm{M} 1 \quad\left(\mathrm{CD} 80^{+}\right)$and $\mathrm{M} 2\left(\mathrm{CD}_{163}{ }^{+}\right)$RAW 264.7 macrophage populations after 1 day of treatment. * Comparison between each biomaterial and control, \# between $\mathrm{CD}^{+}{ }^{+}$and $\mathrm{CD} 163^{+}, \mu$ between nano-HA and nano-SiHA. Statistical significance: \# $\mathrm{p}<0.05$; \#\# $\mathrm{p}<0.01$; ***, $\mu \mu \mu \mathrm{p}<$ 0.005 . 


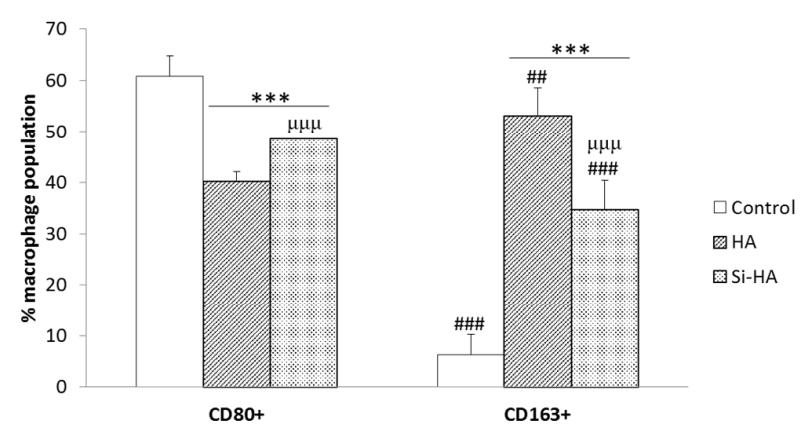

Figure 2. Effects of powdered nano-HA and nano-SiHA (1 $\mathrm{mg} / \mathrm{ml})$ on $\mathrm{M} 1 \quad\left(\mathrm{CD} 80^{+}\right)$and $\mathrm{M} 2\left(\mathrm{CD} 163^{+}\right)$RAW 264.7 macrophage populations after 3 days of treatment. * Comparison between each biomaterial and control, \# between $\mathrm{CD}^{+} 0^{+}$and $\mathrm{CD} 163^{+}, \mu$ between nano-HA and nano-SiHA. Statistical significance: \#\#p $<0.01 ;{ }^{* * *}$, \#\#\#, $\mu \mu \mu \mathrm{p}<0.005$.

The effects of powdered nano-HA and nano-SiHA on M1 $\left(\mathrm{CD}^{+} 0^{+}\right)$and $\mathrm{M} 2\left(\mathrm{CD} 163^{+}\right)$RAW macrophage populations after 3 days of treatment are shown in Figure 2. Control cultures showed a strong increase in the fraction of M1 macrophages and a pronounced decrease of $\mathrm{M} 2$ cells after this longer culture time in the absence of material. This polarization of the control macrophages towards M1 phenotype could be due to the high proliferation rate of these cells under these in vitro conditions after 3 days of culture and it can be related with the role of M1 macrophages in tumor development and growth. It has been shown that M2 macrophages promote tumor growth, while M1 macrophages have tumoricidal action. ${ }^{31}$ At low doses, many anti-cancer therapeutics convert tumor promoting macrophages (M2) to tumoricidal (M1) macrophages in favour of anti-tumor immunity ${ }^{37}$ and subsequent tumor regression. ${ }^{38}$ Thus, the effect observed in the present study with control RAW cells after 3 days of culture can be considered as a regulatory response of these cells in order to control cell growth as an adaptation to microenvironmental conditions and energy demand.

When RAW macrophages were cultured for 3 days in the presence of powdered nano-HA and nano-SiHA, both nanocrystalline hydroxyapatites produced significant $M 1$ decreases and very pronounced $M 2$ increases at this longer time treatment, suggesting a beneficial action of these materials designed for bone repair and bone tissue engineering. Nano-HA exerted significantly higher effects than nano-SiHA on both M1 and M2 RAW populations.

To study the effects of these nanocrystalline hydroxyapatites on a cellular model more similar to the in vivo situation, primary murine macrophages were obtained from the peritoneum of untreated mice and were cultured on either
nano-HA or nano-SiHA disks for 3 days. In order to favour primary macrophage polarization towards either M1 or M2 phenotype and to evaluate the biomaterial effects under these stimulated conditions, either LPS or IL-10 were used as proinflammatory and anti-inflammatory stimuli, respectively. ${ }^{2}$

Figure 3 shows the effects of both materials on the percentage of M1 primary macrophage population with and without proinflammatory stimulus (LPS). Control cultures without material were also carried out in parallel under basal and stimulated conditions.

As it can be observed, the presence of LPS induced the polarization of the control primary macrophages towards a M1 phenotype, in agreement with the action of this stimulus. ${ }^{2}$ Both nano-HA and nano-SiHA disks produced significant M1 decreases under both basal and stimulated conditions, showing a more evident anti-inflammatory effect in the presence of LPS. Under stimulated conditions, the decrease of M1 primary macrophages was more pronounced with nanoSiHA disks than with nano-HA disks (Figure 3).

Figure 4 shows the effects of both materials on the percentage of M2 primary macrophage population with and without antiinflammatory stimulus (IL-10). Control cultures without material were also carried out in parallel under basal and stimulated conditions.

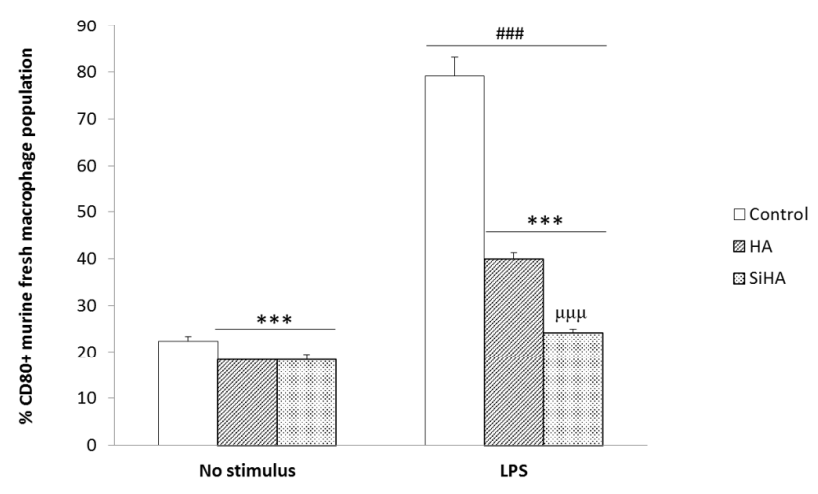

Figure 3. Nano-HA and nano-SiHA effects on $\mathrm{M} 1\left(\mathrm{CD} 80^{+}\right)$ mouse peritoneal macrophage population after 3 days in the presence of pro-inflammatory stimulus (LPS). * Comparison between each biomaterial and control, \# between with and without stimulus, $\mu$ between nano-HA and nano-SiHA. Statistical significance: $* * *, \# \# \#, \mu \mu \mu \mathrm{p}<0.005$. 


\section{ARTICLE}

Journal Name
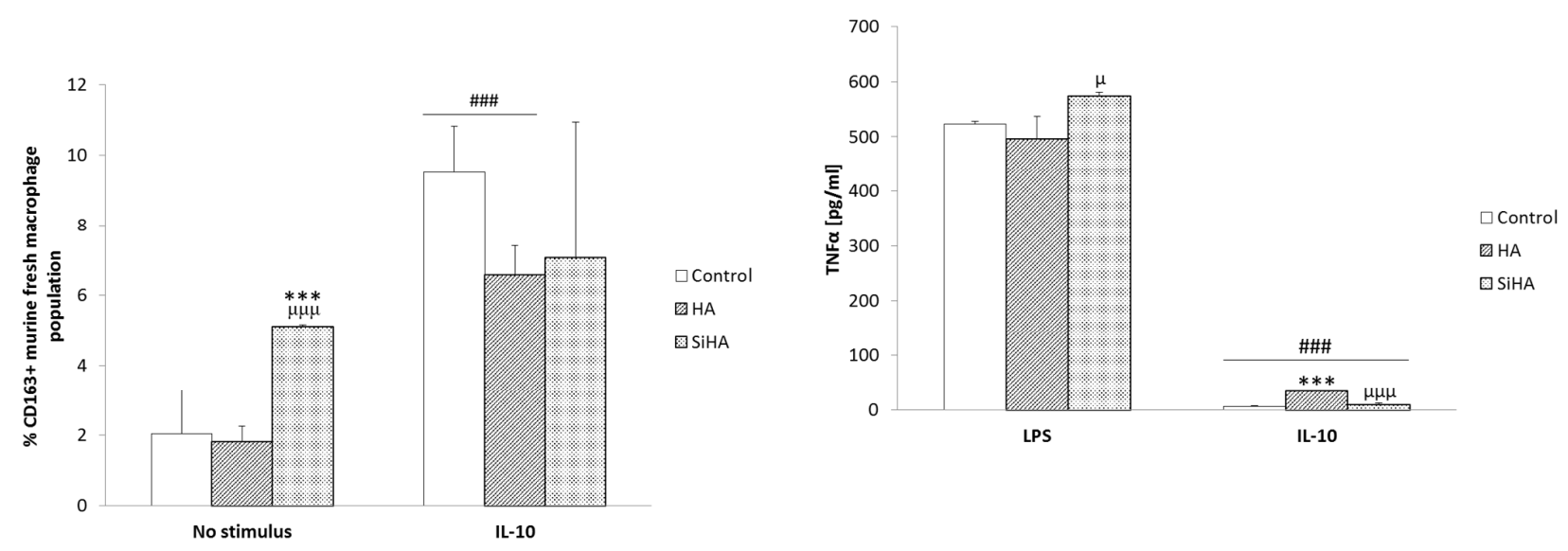

Figure 4. Nano-HA and nano-SiHA effects on $M 2\left(\mathrm{CD}_{163}{ }^{+}\right)$ mouse peritoneal macrophage populations after 3 days in the presence of anti-inflammatory stimulus (IL-10). * Comparison between each biomaterial and control, \# between with and without stimulus, $\mu$ between nano-HA and nano-SiHA. Statistical significance: $* * *, \# \# \#, \mu \mu \mu \mathrm{p}<0.005$.

The polarization of the primary control macrophages towards M2 phenotype was clearly obtained in the presence of IL-10, in agreement with the action of this stimulus. ${ }^{2}$ Although a pronounced increase of $\mathrm{M} 2$ population was observed with primary macrophages cultured on nano-SiHA disks without stimulus, this effect was not induced by nano-HA disks under these basal conditions. In the presence of IL-10, no significant differences were obtained between each material and control under these stimulated conditions (Figure 4).

These studies with primary macrophages indicate that both nano-HA and nano-SiHA disks produced significant M1 decreases under both basal and stimulated conditions. However, the M2 increase was only observed with nano-SiHA disks under basal conditions.

Previous results revealed that both nanocrystalline hydroxyapatites triggered significant interleukin 6 (IL-6) and tumor necrosis factor alpha (TNF- $\alpha$ ) increases in fresh mouse macrophages after 3 days of treatment with these materials. ${ }^{20}$ In the present study, the effects of nano-HA and nano-SiHA disks on the secretion of these two pro-inflammatory cytokines by primary macrophages were evaluated under stimulated conditions in the presence of either LPS (proinflammatory stimulus) or IL-10 (anti-inflammatory stimulus).

As it can be observed in Figures 5 and 6, high levels of both TNF- $\alpha$ and IL- 6 were obtained with or without material in the presence of LPS in comparison with the presence of IL-10, in agreement with the different role of these stimuli.

Figure 5. Nano-HA and nano-SiHA effects on TNF- $\alpha$ secretion by mouse peritoneal macrophages after 3 days in the presence of either pro-inflammatory (LPS) or anti-inflammatory (IL-10) stimuli. * Comparison between each biomaterial and control, \# between LPS and IL-10 stimuli, $\mu$ between nano-HA and nanoSiHA. Statistical significance: $\mu \mathrm{p}<0.05$; $^{* * *}, \# \# \#, \mu \mu \mu \mathrm{p}<$ 0.005 .

Concerning the action of the nanocrystalline hydroxyapatites on TNF- $\alpha$ and IL- 6 levels, only nano-SiHA induced a slight but significant TNF- $\alpha$ increase in the presence of LPS, in comparison with the absence of material (Figure 5). In the presence of IL-10, very low values of both TNF- $\alpha$ and IL- 6 were detected in all cases and only a significant increase of TNF- $\alpha$ (Figure 5) and a slight decrease of IL-6 (Figure 6) were observed with nano-HA in comparison with control cells.

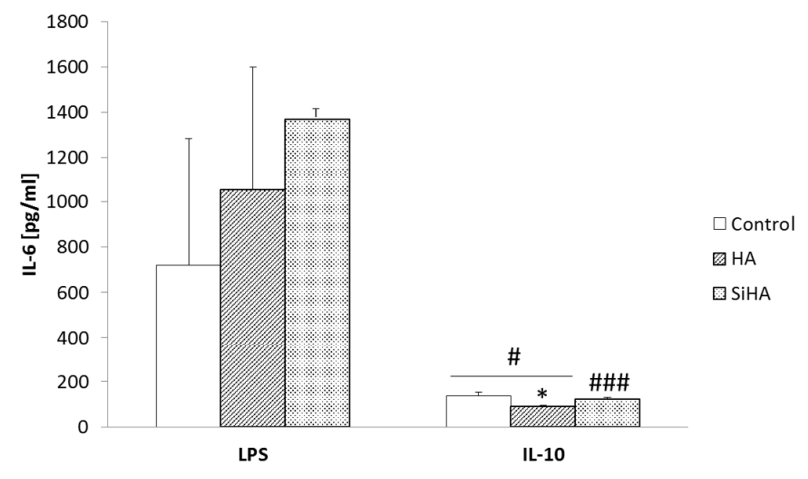

Figure 6. Nano-HA and nano-SiHA effects on IL-6 secretion by mouse peritoneal macrophages after 3 days in the presence of either pro-inflammatory (LPS) or anti-inflammatory (IL-10) stimuli. * Comparison between each biomaterial and control, \# between LPS and IL-10 stimuli. Statistical significance: \#, ${ }^{*}<<$ $0.05 ; \# \# \#<0.005$. 
Since the modulation of macrophage phenotype polarization has been related to alterations in cell shape and a role for the cytoskeleton in the control of macrophage polarization has been suggested [39], a morphological analysis of $\mathrm{M} 1\left(\mathrm{CD} 80^{+}\right)$ and $\mathrm{M} 2\left(\mathrm{CD}_{163^{+}}\right)$cells was carried out by confocal microscopy with primary macrophages cultured either in the absence of material or on disks of both nanocrystalline hydroxyapatites. These morphological studies were performed under stimulated conditions, with either LPS or IL-10, to favour the macrophage polarization towards the $\mathrm{M} 1$ or $\mathrm{M} 2$ phenotype, respectively.

As it can be observed in Figure 7, very different $M 1$ and $M 2$ morphology was observed by confocal microscopy in mouse peritoneal macrophages after 48 hours of treatment with LPS and IL-10. When control primary cells were stimulated with either LPS or IL-10 as pro-inflammatory and anti-inflammatory stimuli, these macrophages polarized towards $\mathrm{M} 1\left(\mathrm{CD} 80^{+}\right)$and M2 $\left(\mathrm{CD} 163^{+}\right)$phenotypes, respectively. This was accompanied by dramatic changes in cell shape, with $M 2$ cells showing an elongated shape compared with $M 1$ cells (Figure 7), in agreement with other authors. ${ }^{39}$

When mouse peritoneal macrophages were cultured on nano$\mathrm{HA}$ and nano-SiHA, disks in the presence of either LPS or IL-10, the characteristic changes in cell shap of $M 1$ and $M 2$ cells were also observed on the biomaterial surfaces (Figure 8). A high number of $\mathrm{M} 2$ cells with elongated shape were observed on nano-SiHA disks in the presence of IL-10 stimulus.
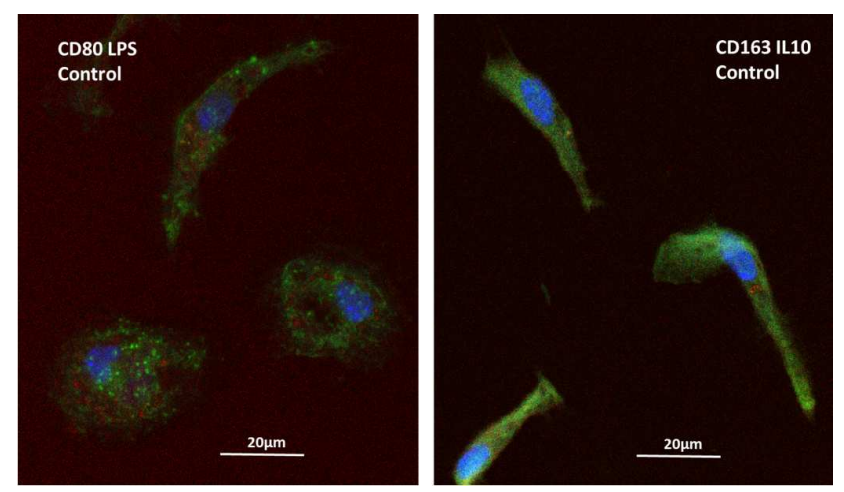

Figure 7. Morphology of $\mathrm{M} 1\left(\mathrm{CD} 80^{+}\right.$, left, red) and $\mathrm{M} 2\left(\mathrm{CD} 163^{+}\right.$, right, red) mouse peritoneal macrophages observed by confocal microscopy after $48 \mathrm{~h}$ of treatment with either proinflammatory (LPS) or anti-inflammatory (IL-10) stimuli. Actin was stained with FITC-Phalloidin (green) and nuclei were stained with DAPI (blue).

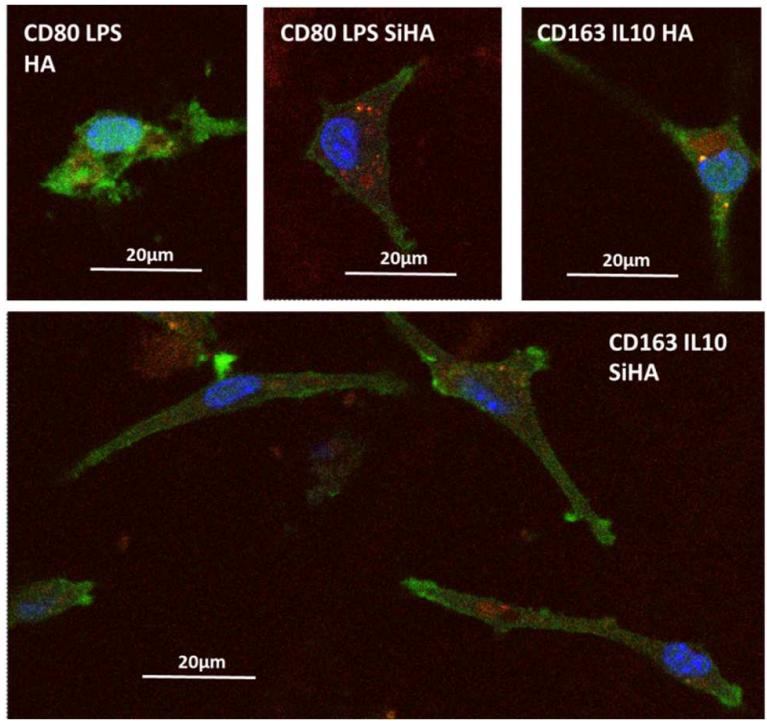

Figure 8. Morphology of $\mathrm{M} 1\left(\mathrm{CD} 80^{+}\right.$, red) and $\mathrm{M} 2\left(\mathrm{CD} 163^{+}\right.$, red $)$ mouse peritoneal macrophages observed by confocal microscopy after $48 \mathrm{~h}$ of treatment with either proinflammatory (LPS) or anti-inflammatory (IL-10) stimuli on either nano-HA or nano-SiHA disks. Actin was stained with FITC-Phalloidin (green) and nuclei were stained with DAPI (blue).

\section{Conclusions}

Although further studies are needed to elucidate the mechanisms underlying polarization of macrophages in response to these nanocrystalline hydroxyapatites, the findings of this study reveal that both nano-HA and nano-SiHA favour the macrophage polarization towards a $M 2$ reparative phenotype, decreasing M1 population. Concerning the response of primary macrophages to the presence of silicon, nano-SiHA produces a more pronounced inhibition than nanoHA on the LPS stimulated polarization towards M1 phenotype and stimulates the polarization towards reparative $M 2$ phenotype in the absence of IL-10. Finally, in the presence of IL-10, the morphological characteristics of primary macrophages seeded onto nano-SiHA indicate that this material stimulates the polarization towards the reparative phenotype to a greater extent than nano-HA. These results reveal that nanocrystalline silicon substituted hydroxyapatites are excellent candidates to ensure an appropriate response in the implantation site of these biomaterials designed for bone repair and bone tissue engineering.

\section{Acknowledgements}

This study was supported by research grants from the Ministerio de Ciencia e Innovación (project MAT2012-35556), Ministerio de Economía y Competitividad (projects MAT201343299-R and PI13/01809) and Agening Network of Excellence (CSO2010-11384-E). M.C. Matesanz is greatly indebted to MEC 
for predoctoral fellowship. The authors wish to thank also to the staff of the Centro de Citometría y Microscopia de Fluorescencia of the Universidad Complutense de Madrid (Spain) for the assistance in the flow cytometry and confocal microscopy studies.

\section{Notes and references}

1 C. A. Janeway and R. Medzhitov, Annu. Rev. Immunol., 2002, 20, 197.

2 D. M. Mosser and J. P. Edwards, Nat. Rev. Immunol., 2008, 8, 958.

3 D. Zhou, C. Huang, Z. Lina, S. Zhan, L. Kong, C. Fang and J. Li, Cell. Signal., 2014, 26, 192.

4 R. Sridharan, A. R. Cameron, D. J. Kelly, C. J. Kearney and F. J. O'Brien, Mater. Today, 2015, 18, 313.

5 A. Mantovani, S. K. Biswas, M. R. Galdiero, A. Sica and M. Locati, J. Pathol., 2013, 229, 176.

6 D. Tugal, X. Liao and M. K. Jain, Arterioscler. Thromb. Vasc. Biol., 2013, 33, 1135.

7 C. Porta, E. Riboldi, A. Ippolito and A. Sica, Semin. Immunol., 2015, 27, 237.

8 K. L. Spiller, S. Nassiri, C. E. Witherel, R. R. Anfang, J. Ng, K. R. Nakazawa, T. Yu and G. Vunjak-Novakovic, Biomaterials, 2015, 37, 194.

9 B. N. Brown, B. D. Ratner, S. B. Goodman, S. Amar and S. F. Badylak, Biomaterials, 2012, 33, 3792.

10 S. V. Dorozhkin, Biomaterials, 2010, 31, 1465.

11 N. Patel, S. M. Best, W. Bonfield, I. R. Gibson, K. A. Hing, E. Damien and P. A. Revell, J. Mater. Sci. Mater. Med., 2002, 13, 1199.

12 M. Vallet-Regí and D. Arcos, J. Mater. Chem., 2005, 15, 1509.

13 A. E. Porter, N. Patel, J. N. Skepper, S. M. Best and W. Bonfield, Biomaterials, 2003, 24, 4609.

14 M. C. Matesanz, M. J. Feito, C. Ramírez-Santillán, R. M. Lozano, S. Sánchez-Salcedo, D. Arcos, M. Vallet-Regí and M. T. Portolés, Macromol. Biosci., 2012, 12, 446.

15 A. Balamurugan, A. H. S. Rebelo, A. F. Lemos, J. H. G. Rocha, J. M. G. Ventura and J. M. F. Ferreira, Dent. Mater., 2008, 24, 1374.

16 D. Arcos, J. Rodríguez-Carvajal and M. Vallet-Regí, Chem. Mater., 2004, 16, 2300.

17 E. M. Carlisle, Calcif. Tissue Int., 1981, 33, 27.

18 S. V. Dorozhkin, Materials, 2009, 2, 1975.

19 E. S. Thian, Z. Ahmad, J. Huang M. J. Edirisinghe, S. N. Jayasinghe, D. C. Ireland, R. A. Brooks, N. Rushton, W. Bonfield and S.M. Best, Acta Biomater., 2010, 6, 750.

20 M. C. Matesanz, M. J. Feito, M. Oñaderra, C. RamírezSantillán, C. da Casa, D. Arcos, M. Vallet-Regí, J. M. Rojo and M. T. Portolés, J. Colloid Interface Sci., 2014, 416, 59.

21 M. J. Feito, R. M. Lozano, M. Alcaide, C. Ramírez-Santillán, D. Arcos, M. Vallet-Regí and M. T. Portolés, J. Mater. Sci. Mater. Med., 2011, 22, 405.

22 M. C. Matesanz, J. Linares, I. Lilue, S. Sánchez-Salcedo, M. J. Feito, D. Arcos, M. Vallet-Regí and M. T. Portolés, J. Mater. Chem. B, 2014, 2, 2910.

23 A. M. Pietak, J. M. Reid, M. J. Stott and M. Sayer, Biomaterials, 2007, 28, 4023.

24 M. Bohner, Biomaterials, 2009, 30, 6403.

25 M. I. Kay, R. A. Young and A. S. Posner, Nature, 1964, 204, 1050.

26 J. Rodríguez-Carvajal, Physica B, 1993, 192, 55.

27 B. B. Mishell and J. M. Shiigi, in: B. B. Mishell and J. M. Shiigi, editors. Selected Methods in Cellular Immunology. San Francisco: Freeman WH \& Co 1980, p 1-28.
28 S. E. Burastero, Z. Magnani, C. Confetti, L. Abbruzzese, S. Oddera, P. Balbo, G. A. Rossi and E. Crimi, J. Allergy Clin. Immunol., 1999, 103, 1136.

29 J. B. Patton, R. R. Rowland, D. Yoo and K. O. Chang, Virus Res., 2009, 140, 161.

30 F. Porcheray, S. Viaud, A. C. Rimaniol, C. Leone, B. Samah, N. Dereuddre-Bosquet, D. Dormont, and G. Gras, Clin. Exp. Immunol., 2005, 142, 481.

31 A. Sica and A. Mantovani, J. Clin. Invest., 2012, 122, 787.

32 D. M. Mosser, J. Leukoc. Biol., 2003, 73, 209.

33 F.O. Martinez and S. Gordon, F1000Prime Rep., 2014, 6, 13.10.12703/P 6 .

34 A. Mantovani, A. Sica, S. Sozzani, P. Allavena, A. Vecchi and M. Locati, Trends Immunol., 2004, 25, 677.

35 G. Solinas, G. Germano, A. Mantovani and P. Allavena, J. Leukoc. Biol., 2009, 86, 1065.

36 S. Gordon, Nat. Rev. Immunol., 2003, 3, 23.

37 K. Bryniarski, M. Szczepanik, M. Ptak, M. Zemelka and W. Ptak, Pharmacol. Rep., 2009, 61, 550.

38 L. Bracci, G. Schiavoni, A. Sistigu and F. Belardelli, Cell Death Differ., 2014, 21, 15.

39 F. Y. McWhorter, T. Wang, P. Nguyen, T. Chung and W. F. Liu, Proc. Natl. Acad. Sci. U. S. A., 2013, 110, 17253. 


\section{nano-HA and nano-SiHA hydroxyapatites}

M1 macrophage

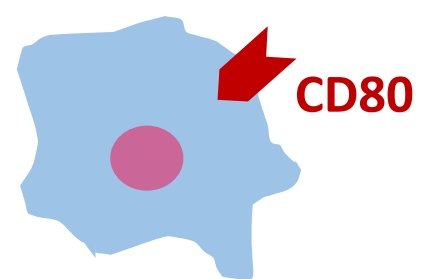

Proinflammatory phenotype
M2 macrophage

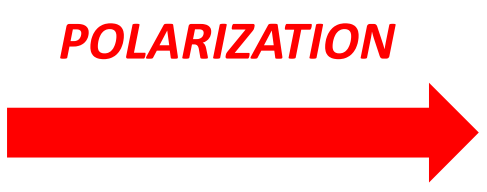

Reparative phenotype 